\title{
Physical activity and cancer: A cross-sectional study on the barriers and facilitators to exercise during cancer treatment
}

\author{
by Stephanie Fernandez, Jenna Franklin, Nafeesa Amlani, Christian DeMilleVille, Dana Lawson, and Jenna Smith
}

\begin{abstract}
The purpose of this investigation was to explore the barriers and facilitators to exercise in individuals with cancer in Ontario. A cross-sectional study using an online survey was used to collect qualitative and quantitative data. A convenience sample was used to recruit individuals with a current or previous diagnosis of any type of cancer. Percentages and themes were obtained from the data.
\end{abstract}

Data were collected from 30 participants of which 63.3\% reported infrequent, low intensity physical activity during treatment. Barriers to exercise during treatment included physical symptoms and lack of awareness of exercise programs. Facilitators for exercise were past positive experience with exercise and accessibility. More than $80 \%$ of participants did not receive education on the importance of exercise.
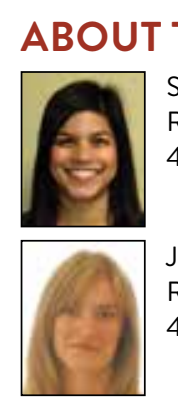

Jenna Franklin, MSc(PT), McMaster University School of Rehabilitation Sciences, Institute for Applied Health Sciences, Rm. 402, 1400 Main St. W, Hamilton, ON L8S 1 C7

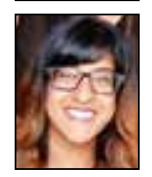

Nafeesa Amlani, MSc(PT), McMaster University School of Rehabilitation Sciences, Institute for Applied Health Sciences, Rm. 402, 1400 Main St. W, Hamilton, ON L8S 1 C7

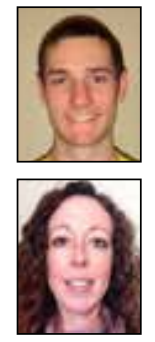

Christian DeMilleVille, MSc(PT), McMaster University School of Rehabilitation Sciences, Institute for Applied Health Sciences, Rm. 402, 1400 Main St. W, Hamilton, ON L8S 1 C7

Dana Lawson, HBKin, Physio-Care Services, 845 Upper James Street, \#208, Hamilton, ON L9C 3A3

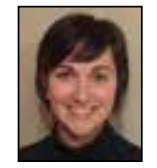

Jenna Smith, MSc(PT), McMaster University School of Rehabilitation Sciences, Institute for Applied Health Sciences, Rm. 402, 1400 Main St. W, Hamilton, ON L8S 1C7; PhysioCare Services, 845 Upper James Street, \#208, Hamilton, ON L9C 3 A3

Corresponding author: Jenna Smith, McMaster University School of Rehabilitation Sciences, Institute for Applied Health Sciences, Rm. 403, 1400 Main St. W, Hamilton, ON L8S 1C7. Email: jenna.smith@shaw.ca

Phone: 905-869-0599; Fax: 905-524-0069

DOI: $10.5737 / 236880762513742$
The findings of this study should encourage health care professionals working in oncology to provide patient education on the importance of exercise.

Key points:

- Two-thirds of the individuals decreased their exercise level during treatment.

- The prevalence barriers to exercise during treatment were fatigue and lack of knowledge about benefits of exercise and existing programs.

- Cancer patients would benefit from thorough and consistent education about exercise during cancer treatment.

\section{INTRODUCTION}

ancer is the leading cause of death in Canada (Canadian Cancer Society, 20I3). The disease and its treatments are projected to cost the Canadian health care system $\$$ I77.5 billion over the next 30 years (Smetanin \& Kobak, 2005). There are several side effects that arise from both cancer and its treatments. A major one is cancer-related fatigue. This is defined as a persistent, subjective sense of physical, emotional or cognitive tiredness (Madden \& Newton, 2006). Further reported side effects include pain, as well as cardiovascular, neurological, endocrine, musculoskeletal and other system changes (Schmitz et al., 2010).

Exercise has been shown to decrease the various side effects of cancer and its related treatment, independent of type or stage of cancer (Velthuis, Agasi-Idenburg, Aufdemkampe, \& Wittink, 20IO; Knols, Aaronson, Uebelhart, Fransen, \& Aufdemkampe, 2005). According to a recent Cochrane Review, exercise has demonstrated significant positive effects on health-related quality of life in those with cancer during active treatment, with greater improvements seen with moderate to vigorous intensities (Mishra et al., 20I2). Additionally, varying modes of exercise exert positive effects on physiological outcomes across cancer types, including a reduction in pain and nausea, as well as an increase in strength and shoulder range of motion (Knols et al., 2005). The benefits of exercise also extend to domains of mental health, such as improvements of depressive symptoms in cancer survivors (Craft, Vanlterson, Helenowski, Rademaker, \& Courneya, 20II).

Despite the established benefits to exercise, only $22 \%$ of Canadian cancer survivors were found to be physically active, which was defined as walking approximately one hour per day (Courneya, Katzmarzyk, \& Bacon, 2008). Fatigue and deconditioning, both of which are common side effects of treatment, can present as physical barriers that may lead to social isolation 
and lack of routine exercise (Blaney et al., 20Io). Additionally, Blaney et al. (2010) found that perceived environmental and financial barriers, such as non-specific exercise programs and high costs, can contribute towards decreased levels of physical activity (PA). In a recent cross-sectional study conducted in the United Kingdom, the top reported barriers to exercise participation were found to be joint stiffness, fatigue and pain, all of which may be lessened by routine engagement in PA (Blaney, Lowe-Strong, Rankin-Watt, Campbell, \& Gracey, 20I3). A lack of formal education regarding cancer and exercise has also been cited as a barrier (Blaney et al., 2013). Alternatively, the following factors have been shown by Blaney et al. (2013) to have potential in facilitating participation in exercise: an enjoyable and varied program, approval from a health care professional, and individualized goals and treatment interventions. However, variation does occur in the literature and it appears that specific facilitators depend on gender, type of cancer, and other individual variations.

Although the literature has reported multiple benefits of exercise for persons with cancer, few are participating in regular PA (Cheifetz, 20I3). The bulk of this research, however, has focused primarily on breast cancer or has been conducted outside of Ontario (Canestraro et al., 2013). The purpose of this study was to explore the barriers and facilitators to exercise in individuals with cancer in Ontario who are currently receiving or have undergone treatment. A secondary purpose was to explore if these barriers and facilitators differed depending on cancer type. It was hypothesized that: (I) barriers will include pain, fatigue and other physical symptoms such as weakness or nausea, as well as a lack of knowledge about existing programs and the benefits of exercise; (2) facilitators will include previous engagement in PA, and education on available programs and the benefits of exercise.

\section{METHODS}

\section{Design and questionnaire}

This was a cross-sectional study using an anonymous online survey for quantitative and qualitative data collection. A 23-item survey was created and administered through SurveyMonkey. The survey was compiled by integrating relevant research with the clinical experience of the investigators. Informed consent was required to access the survey. Participants completed the survey at home, in the clinic of the primary investigators or at a preferred location. The survey required Io-20 minutes to complete. The questions addressed exercise frequency and intensity prior to diagnosis, as well as during and after cancer treatment. Perceived barriers and facilitators to exercise were also investigated, in addition to health care providers' delivery of exercise-related information. For the purpose of this study, the terms exercise and PA were used interchangeably. Ethical approval was granted by the McMaster Research Ethics Board in February 20I3.

\section{Participants}

A convenience sample of individuals with cancer who were participating in an outpatient physiotherapy program or established cancer support organizations in communities within Ontario were recruited for this study. Individuals who met the inclusion criteria were those living in Ontario, over the age of I8, with a current or previous diagnosis of any type of cancer, and people either undergoing or having already received treatment for cancer. No eligibility criteria were stated in relation to how long the participant had to be finished their treatment. All people were asked to recall their behaviour during cancer treatment. Participants were identified by their health care professional and asked by these professionals to participate in the study.

\section{Data collection and analysis}

Data were collected over a period of three months and interpreted quantitatively with supplemental qualitative content methodology. The closed-ended questions were analyzed quantitatively by calculating percentages. The open-ended questions were interpreted using qualitative content analysis. This involves the scanning and coding of information into distinct meaningful categories, from which themes are derived (Hsieh \& Shannon, 2005). This interpretation is subjective in nature and preconceived notions surrounding predicted themes are not imposed (Hsieh \& Shannon, 2005). Two of the investigators (JF and SF) independently reviewed, interpreted, and organized the data into themes. Findings were discussed between these investigators, and again with the primary investigator, until a consensus was reached. Participants were not required to answer all of the questions. In order to have the survey included in the study, $>85 \%$ of questions must have been answered. Response patterns to each question provided by the entire sample were reviewed, followed by an analysis of individual responses.

\section{RESULTS}

\section{Participant characteristics}

A total of 30 individuals with a current or past diagnosis of cancer completed the survey. Details of participant demographics can be found in Table I. All but two participants were female $(93.1 \%)$ and the mean age at diagnosis was 47.8 years $(\mathrm{SD}=+/-\mathrm{I} 5.8)$. The most common diagnosis was breast cancer (58.6\%). Of the sample, a diagnosis of Stage I (63.2\%) and Stage II (2I.0\%) cancer was most commonly identified. Almost the entire sample (96.7\%) indicated that some type of

Table 1: Participants' demographic information

\begin{tabular}{|l|l|l|l|l|l|l|l|l|l|l|l|l|}
\hline Demographics & Sex & \multicolumn{4}{l|}{ Age } & \multicolumn{2}{l|}{ Cancer type } & \multicolumn{2}{l|}{ Cancer stage } & \multicolumn{3}{l|}{ Treatment received } \\
\hline & Female & $\mathbf{5} \mathbf{5 0}$ & $\mathbf{> 5 0}$ & Breast & Other & $\mathbf{1}$ & $\mathbf{2}$ & $\mathbf{3}$ & $\mathbf{4}$ & Chemotherapy & Radiation & Surgery \\
\hline $\mathrm{N}$ & 27 & 15 & 16 & 17 & 12 & 12 & 4 & 1 & 2 & 21 & 14 & 23 \\
\hline$\%$ & 93.1 & 48.3 & 51.7 & 58.6 & 41.4 & 63.2 & 21 & 5.3 & 10.5 & 72.4 & 48.3 & 79.3 \\
\hline
\end{tabular}


Table 2: Participants' physical activity levels

\begin{tabular}{|c|c|c|c|c|c|c|c|c|c|c|c|c|c|c|c|}
\hline \multirow{2}{*}{$\begin{array}{l}\text { Activity } \\
\text { Level }\end{array}$} & \multicolumn{4}{|c|}{ Frequency } & \multicolumn{11}{|c|}{ Intensity } \\
\hline & Frequent & Moderate & Occasional & Infrequent & 0 & 1 & 2 & 3 & 4 & 5 & 6 & 7 & 8 & 9 & 10 \\
\hline
\end{tabular}

Prior to Cancer Diagnosis

\begin{tabular}{|l|c|c|c|c|c|c|c|c|c|c|c|c|c|c|c|}
\hline $\mathrm{N}$ & 2 & 11 & 12 & 5 & 3 & 1 & 6 & 0 & 2 & 11 & 3 & 2 & 2 & 0 & 0 \\
\hline$\%$ & 6.7 & 36.7 & 40 & 16.7 & 10 & 3.3 & 20 & 0 & 6.7 & 36.7 & 10 & 6.7 & 6.7 & 0 & 0 \\
\hline
\end{tabular}

During Cancer Treatment

\begin{tabular}{|l|c|c|c|c|c|c|c|c|c|c|c|c|c|c|c|}
\hline $\mathrm{N}$ & 0 & 6 & 5 & 19 & 19 & 0 & 4 & 1 & 1 & 5 & 0 & 0 & 0 & 0 & 0 \\
\hline$\%$ & 0 & 20 & 16.7 & 63.3 & 63.3 & 0 & 13.3 & 3.3 & 3.3 & 16.7 & 0 & 0 & 0 & 0 & 0 \\
\hline
\end{tabular}

After Cancer Treatment

\begin{tabular}{|l|c|c|c|c|c|c|c|c|c|c|c|c|c|c|c|}
\hline $\mathrm{N}$ & 3 & 12 & 10 & 3 & 1 & 1 & 8 & 0 & 1 & 10 & 2 & 1 & 4 & 0 & 0 \\
\hline$\%$ & 10.7 & 42.9 & 35.7 & 10.7 & 3.6 & 3.6 & 28.6 & 0 & 3.6 & 35.7 & 7.1 & 3.6 & 14.3 & 0 & 0 \\
\hline
\end{tabular}

*Frequency levels: Frequent (6-7x/week), Moderate (3-5x/week), Occasional (1-2x/week), Infrequent $(<1 x /$ week)

** Intensity levels: 0 (very low intensity [e.g., very slow walk]), 2 (low intensity [e.g., normal walk]), 5 (moderate intensity [e.g., fast paced walk, bike ride, or activity where sweating by the end]), 8 (high intensity [e.g., run, increased heart rate, sweating and heavy breathing]), 10 (very intense [e.g., marathon training]).

cancer-related treatment had been received, with surgery being the most commonly reported (79.3\%), followed by chemotherapy (72.4\%).

\section{Physical activity status}

Participants were asked about PA prior to, during and after cancer treatment, the results of which are presented in Table 2. Preceding cancer diagnosis, the majority of participants indicated occasional (40\%, I-2x/week) or moderate $(36.7 \%, 3-5 \mathrm{x}$ / week) frequency of PA. Moderate intensity PA was most commonly reported (36.7\%), which was described as a fast-paced activity where one is sweating by the end.

During cancer treatment, the majority of the sample (63.3\%) reported engagement in infrequent activity (<Ix/week) at a very low intensity, described as a very slow walk. Only a small number of individuals (I6.7\%) maintained moderate intensity PA during treatment. Participants with breast cancer were more likely to participate in moderate intensity exercise $(23.5 \%)$, compared to those with another form of cancer (I6.7\%).

Following treatment, moderate $(42.9 \%)$ and occasional (35.7\%) PA was most common. The intensity that the majority reported was moderate $(35 \cdot 7 \%)$. When asked what influenced a change in PA level, the most common reasons were that participants were feeling better (65.4\%) and had more energy (61.5\%). Following treatment, a higher percentage of patients with breast cancer (92.8\%) increased their PA level compared to those with other forms of cancer $(80 \%)$.

\section{Written comments}

Open-ended questions were asked in regards to the type of PA individuals engaged in prior to cancer diagnosis and during cancer treatment. Common themes, with respect to type of

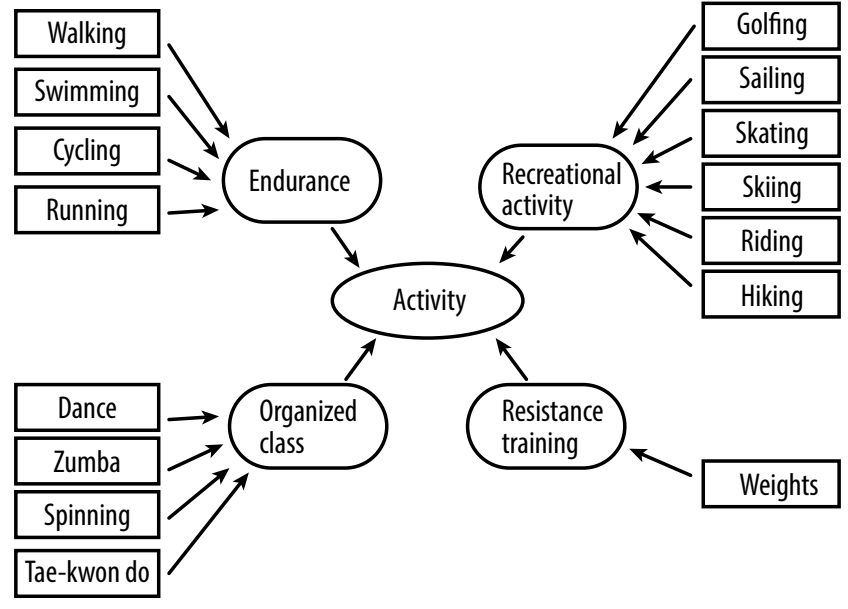

Figure I: Common themes of exercise type prior to cancer diagnosis

exercise prior to cancer diagnosis, were endurance exercise, resistance training, various recreational activities, and organized exercise classes. (Refer to Figure I for a visual representation of these themes.) Endurance exercise was reported most frequently and included walking, swimming, cycling, and running. Recreational activities, organized exercise classes, and resistance training were also reported.

During treatment, endurance activity was again the most popular. The majority reported walking as the preferred mode of activity. The theme of organized exercise classes was also found during treatment. These included yoga classes and formal programs offered by centres such as Wellspring and the YMCA. (See Figure 2.) 


\section{Barriers to exercise and side effects from treatment}

Participants were asked about barriers to exercise during cancer treatment and the top three reported were lack of energy (8I.5\%), physical symptoms (74.1\%), and unawareness of available programs (5I.9\%). Other barriers included physical challenges, belief that it would make them feel worse, and a lack of awareness that they should be exercising. (Refer to Table 3.) When comparing the results by cancer type, individuals with a diagnosis other than breast cancer reported improved energy levels (lack of energy was described 66.7\% of the time with these individuals compared to $82.4 \%$ of the time for participants with breast cancer), but an increased lack of awareness about available exercise programs (50\% versus $4 \mathrm{I} .2 \%)$.

The three most commonly reported side effects from cancer treatment included fatigue (89.7\%), numbness and/or tingling in extremities (69.0\%), and muscle weakness (65.5\%). Other side effects included nausea (51.7\%), pain $(48.3 \%)$, depression (48.3\%), anxiety (4I.4\%), limited joint movement $(27.6 \%)$, and vomiting (24.1\%). The majority of participants $(76.7 \%)$ indicated that these physical symptoms influenced their ability or desire to exercise. When comparing the results across cancer type, participants with breast cancer reported higher levels of muscle weakness (70.6\%) and less numbness and tingling in their extremities (58.8\%).

\section{Facilitators to exercise during treatment}

The top three facilitators during treatment were previous positive experience with exercise $(63.2 \%)$, exercise prior to cancer treatment with a desire to maintain this activity $(47.4 \%)$,

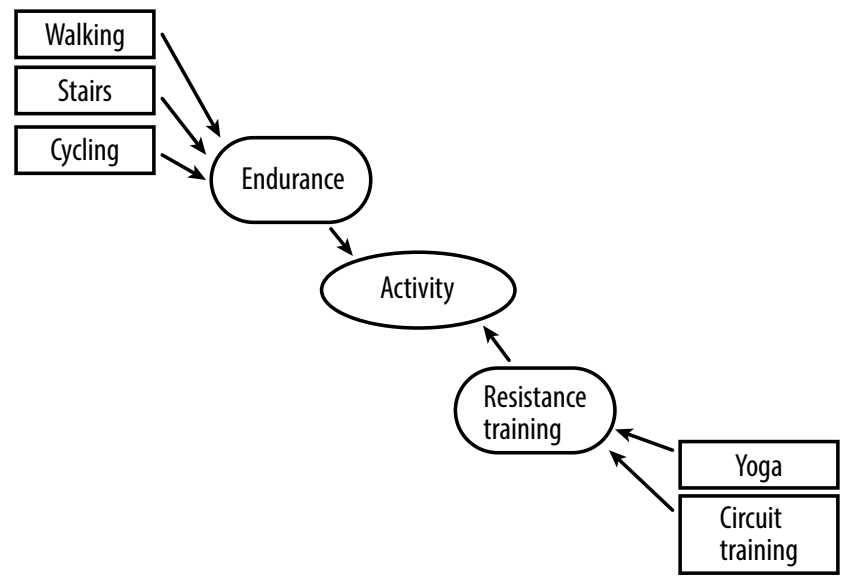

Figure 2: Common themes of exercise type during cancer treatment and accessible exercise services (42.1\%). Additional facilitators were that participants were told that exercise was good for them, friends or family participated in exercise with them, and more free time to exercise. (Refer to Table 4.) When comparing across cancer types, having a past positive experience with exercise was a much stronger facilitator for individuals with breast cancer $(75 \%)$ then for those with other forms of cancer (25\%).

\section{Education}

Participants were also asked about the presence and type of education provided by health care professionals related to PA during cancer treatment. (Refer to Table 5.) A larger portion of the participants with breast cancer (58.8\%) reported that they were aware of the importance of exercise versus those with a different form of cancer (33.3\%). Overall, 83.3\% of oncologists and $86.7 \%$ of nurses did not educate these patients on the importance of exercise throughout their cancer treatment. Furthermore, 66\% reported that general information or instruction on PA was not provided by anyone during treatment.

\section{DISCUSSION}

The results of this study demonstrate the influence of cancer and cancer-related treatment on the ability of patients to exercise, as well as the importance of education regarding PA in promoting exercise in this population. Prior to diagnosis, I7\% of participants engaged in infrequent PA. This number increased to $66.7 \%$ during treatment. On an individual level, the majority (two thirds) of participants dropped a level in relation to the frequency of physical activity (for example, frequent to moderate or moderate to infrequent) during cancer treatment. Following cessation of treatment, it was found that the majority of individuals returned to pre-diagnosis activity status, a pattern that was consistent among all types of cancer.

Data collected from this study support previous research showing that prevalent barriers to exercise during treatment include fatigue and a lack of knowledge on existing programs and the benefits of exercise. Furthermore, physical side effects of treatment had a negative influence on activity levels, regardless of cancer type. This finding is in accordance with other barriers to exercise previously reported in the literature (Sander, Wilson, Izzo, Mountford, \& Hayes, 20I2; Brunet, Taran, Burke, \& Sabiston, 2013).

More than half of the participants in our study reported being unaware of the benefits of exercise for cancer-related

Table 3: Barriers to exercise participation

\begin{tabular}{|l|c|c|c|c|c|c|c|}
\hline Barriers & Lack of Energy & $\begin{array}{c}\text { Physical } \\
\text { Symptoms }\end{array}$ & $\begin{array}{c}\text { Unawareness } \\
\text { of Programs }\end{array}$ & $\begin{array}{c}\text { Physical } \\
\text { Challenges }\end{array}$ & $\begin{array}{c}\text { Poor Perceptions } \\
\text { of Exercise }\end{array}$ & $\begin{array}{c}\text { Unawareness of Need } \\
\text { to Exercise }\end{array}$ & 6 \\
\hline N & 22 & 20 & 14 & 10 & 7 & 6 \\
\hline$\%$ & 81.5 & 74.1 & 51.9 & 37 & 25.9 & 22.2 \\
\hline
\end{tabular}


Table 4: Facilitators to exercise participation

\begin{tabular}{|l|l|l|l|l|l|l|l|}
\hline Facilitators & $\begin{array}{l}\text { Positive Previous } \\
\text { Experience with Exercise }\end{array}$ & $\begin{array}{l}\text { Maintain } \\
\text { Fitness Levels }\end{array}$ & $\begin{array}{l}\text { Accessible Exercise } \\
\text { Programming }\end{array}$ & $\begin{array}{l}\text { Positive } \\
\text { Perceptions of } \\
\text { Exercise }\end{array}$ & $\begin{array}{l}\text { Encouragement } \\
\text { from Important } \\
\text { Others }\end{array}$ & $\begin{array}{l}\text { Time } \\
\text { Allowance }\end{array}$ & $\begin{array}{l}\text { Other } \\
\text { N }\end{array}$ \\
\hline$\%$ & 63.2 & 9 & 8 & 7 & 6 & 5 & 9 \\
\hline
\end{tabular}

Table 5: Education related to physical activity during cancer treatment

\begin{tabular}{|l|c|c|c|}
\hline Education provided by: & Oncologist & Primary nurse & Anyone \\
\hline Yes & 5 & 4 & 12 \\
\hline No & 25 & 26 & 18 \\
\hline
\end{tabular}

symptoms. Four individuals in particular indicated that they had received education about PA, but were unfamiliar with the positive influences of exercise. This lack, or disconnect, of information provided to patients is a common theme throughout the literature (Cheifetz, 20I3; Ottenbacher et al., 20II). In a qualitative study, Sander et al. (20I2) found that patients with breast cancer reported a fear of exercising because they did not know which exercises were safe to perform. Our findings, therefore, highlight the importance of thorough and consistent education for patients with cancer to decrease apprehension towards exercise. Finally, respondents in our study were unaware of available exercise programs. Thus, health care professionals should consider integrating information regarding exercise programming and community resources into their usual counselling (Jones $\&$ Courneya, 2002).

The results of this study also bring forward novel information when comparing barriers and facilitators to exercise by cancer type. When comparing participants with breast cancer to all other forms of cancer, those participants with breast cancer reported greater levels of fatigue and muscle weakness than those with other cancer types. This finding is not surprising, as treatment regimens and medications used for chemotherapy differ by cancer type and cause different side effects. Another important finding was that those with breast cancer were more often educated about the importance of PA during cancer treatment by a health care professional and, hence, were more aware of the need to exercise during cancer treatment. This finding may be a result of the greater amount of literature available stating the benefits of exercise for individuals with breast cancer compared to the available literature for other forms of cancer. The treatment protocols for various forms of cancer may also be a contributor to this finding, as those with breast cancer are often involved with a multidisciplinary team early in the course of their treatment.

\section{Clinical implications}

The results of this study provide implications for the incorporation of exercise programs for individuals undergoing treatment for cancer. Specifically, the results note the importance of patient education regarding PA and the reduction of barriers related to physical symptoms early in treatment. Many health care professionals (for example physiotherapists and nurses) are equipped with the knowledge to prescribe exercise and provide education on this topic, however, their services are currently underutilized in cancer rehabilitation (Cheifetz, 2013).

\section{LIMITATIONS}

Limitations to this study should be taken into account when considering the results. First, our small and predominantly female sample may introduce difficulty in generalizing the results to a larger population. Also, the presence of unknown comorbidities or confounding variables in this population could have influenced the results. Finally, the data included in the study were collected retrospectively, which may introduce recall bias.

\section{CONCLUSION}

This study confirmed that individuals' exercise levels decrease when patients are undergoing treatment for cancer. As hypothesized, fatigue, other physical symptoms and lack of knowledge regarding exercise and its benefits were the most commonly reported barriers to exercise participation. Pain was also found to be a contributor. Our hypothesis of previous engagement in exercise being a facilitator was supported. Other facilitators were past positive experience with exercise and accessibility of exercise services. Furthermore, our results were in accordance with the available literature on this topic and reinforce the importance of the role of health care professionals in cancer rehabilitation. Of interest was the differences found in results when comparing breast cancer to other forms of cancer. Patients with breast cancer reported higher levels of fatigue and muscle weakness, as well as higher levels of education and knowledge on the need to exercise during cancer treatment.

\section{ACKNOWLEDGEMENTS}

The authors would like to thank Wellspring Cancer Support Network and the Can Well Program for their assistance in recruiting participants. 


\section{REFERENCES}

Blaney, J.M., Lowe-Strong, A., Rankin, J., Campbell, A., Allen, J., \& Gracey, J. (2010). The cancer rehabilitation journey: Barriers to and facilitators of exercise among patients with cancer-related fatigue. Physical Therapy, 90, II35-47.

Blaney, J.M., Lowe-Strong, A., Rankin-Watt, J., Campbell, A., \& Gracey, J.H. (2013). Cancer survivors' exercise barriers, facilitators and preferences in the context of fatigue, quality of life and physical activity participation: A questionnaire-survey. Psychooncology, 22, I86-94.

Brunet, J., Taran, S., Burke, S., \& Sabiston, C.M. (2013). A qualitative exploration of barriers and motivators to physical activity participation in women treated for breast cancer. Disability and Rehabilitation, 24, 2038-2045.

Canadian Cancer Society's Advisory Committee on Cancer Statistics. (2013). Canadian Cancer Statistics 2013. Retrieved from http:// www.cancer.ca/ /media/cancer.ca/CW/cancer\%2oinformation/ cancer\%20I0I/Canadian\%2ocancer\%2ostatistics/canadiancancer-statistics-2OI3-EN.pdf

Canestraro, A., Nakhle, A., Stack, M., Strong, K., Wright, A., Beauchamp, M., et al. (20I3). Oncology rehabilitation provision and practice patterns across Canada. Physiotherapy Canada, ${ }_{5}$, 94-IO2.

Cheifetz, O. (2013). Clinicians commentary on Sing et al. Physiotherapy Canada, 65, 192-3.

Courneya, KS., Katzmarzyk, PT. \& Bacon, E. (2008). Physical activity and obesity in Canadian cancer survivors. Cancer, 112, 2475-82.

Craft, L.L., Vanlterson, E.H., Helenowski, I.B., Rademaker, A.W., \& Courneya, K.S. (20II). Exercise effects on depressive symptoms in cancer survivors: A systematic review and meta-analysis. Cancer Epidemiology, Biomarkers and Prevention, 21, 3-19.

Hsieh, H.F., \& Shannon, S.E. (2005). Three approaches to qualitative content analysis. Qualitative Health Research, 15, I277-88.

Jones, L.W., \& Courneya, K.S. (2002). Exercise counseling and programming preferences of cancer survivors. Cancer Practice, 10, 208-I5.
Knols, R., Aaronson, N.K., Uebelhart, D., Fransen, J., \& Aufdemkampe, G. (2005). Physical exercise in cancer patients during and after medical treatment: A systematic review of randomized and controlled clinical trials. Journal of Clinical Oncology, 23, 3830-3842.

Madden, J., \& Newton, S. (2006). Why am I so tired all the time? Understanding cancer-related fatigue. Clinical Journal of Oncology Nursing, 10(5), 659-66I.

Mishra, S.I., Scherer, R.W., Geigle, P.M., Berlanstein, D.R., Topaloglu, O., Gotay, C.C., et al. (2OI2). Exercise interventions on healthrelated quality of life for cancer survivors. Cochrane Database of Systematic Reviews, 8, CDoo7566.

Ottenbacher, A.J., Day, R.S., Taylor, W.C., Sharma, S.V., Sloane, R., Snyder, D.C., et al. (20II). Exercise among breast and prostate cancer survivors-What are their barriers? Journal of Cancer Survivorship, 5.4, 413-I9.

Sander, A.P., Wilson, J., Izzo, N., Mountford, S.A., \& Hayes, K.W. (2OI2). Factors that affect decisions about physical activity and exercise in survivors of breast cancer: A qualitative study. Physical Therapy, 92, 525-36.

Schmitz, K.H., Courneya, K.S., Matthews, C., Demark-Wahnefried, W., Galvao, D.A., Pinto, B.M, et al. (2010). American College of Sports Medicine roundtable on exercise guidelines for cancer survivors. Medicine \& Science in Sports a Exercise, 42(7), I409-I426.

Smetanin, P., \& Kobak, P. (2005). Interdisciplinary Cancer Risk Management: Canadian Life and Economic Impacts. Risk Analytica.

Velthuis, M.J., Agasi-Idenburg, S.C., Aufdemkampe, G., \& Wittink, H.M. (20IO). The effect of physical exercise on cancer-related fatigue during cancer treatment: A meta-analysis of randomised controlled trials. Clinical Oncology, 22(3), 208-22I. 